TAIWANESE JOURNAL OF MATHEMATICS

Vol. 23, No. 6, pp.1303 1315 December 2019

DOI: $10.11650 / \mathrm{tjm} / 190105$

\title{
Enumerations of Permutations by Circular Descent Sets
}

\author{
Hungyung Chang, Jun Ma* and Jean Yeh
}

\begin{abstract}
The circular descent of a permutation $\sigma$ is a set $\{\sigma(i) \mid \sigma(i)>\sigma(i+1)\}$. In this paper, we focus on the enumerations of permutations by the circular descent set. Let $\operatorname{cdes}_{n}(S)$ be the number of permutations of length $n$ which have the circular descent set $S$. We derive the explicit formula for $\operatorname{cdes}_{n}(S)$. We describe a class of generating binary trees $T_{k}$ with weights. We find that the number of permutations in the set $\operatorname{CDES}_{n}(S)$ corresponds to the weights of $T_{k}$. As a application of the main results in this paper, we also give the enumeration of permutation tableaux according to their shape.
\end{abstract}

\section{Introduction}

Throughout this paper, let $[m, n]:=\{m, m+1, \ldots, n\},[n]:=[1, n]$ and $\mathfrak{S}_{n}$ be the set of all the permutations in the set $[n]$. We will write permutations $\sigma$ of $\mathfrak{S}_{n}$ in the form $\sigma=(\sigma(1) \sigma(2) \cdots \sigma(n))$. We say that a permutation $\sigma$ has a circular descent of value $\sigma(i)$ if $\sigma(i)>\sigma(i+1)$. The circular descent set of a permutation $\sigma$, denoted $\operatorname{CDES}(\sigma)$, is the set $\{\sigma(i) \mid \sigma(i)>\sigma(i+1)\}$. For example, the circular descent set of $\sigma=(48632517)$ is $\{8,6,5,3\}$. For any $S \subseteq[n]$, we define a set $\operatorname{CDES}_{n}(S)$ as $\operatorname{CDES}_{n}(S)=\left\{\sigma \in \mathfrak{S}_{n} \mid\right.$ $\operatorname{CDES}(\sigma)=S\}$ and use $\operatorname{cdes}_{n}(S)$ to denote the number of the permutations in the set $\operatorname{CDES}_{n}(S)$, i.e., $\operatorname{cdes}_{n}(S)=\left|\operatorname{CDES}_{n}(S)\right|$. First, for any $n \geq 2$ and $S \subseteq[n]$, we prove that the necessary and sufficient condition for $\operatorname{CDES}_{n}(S) \neq \emptyset$ is $1 \notin S$. The main results in this paper are as follows:

Theorem 1.1. Suppose that $S=\left\{s_{1}, s_{2}, \ldots, s_{k}\right\} \subseteq[n]$ with $s_{1}>s_{2}>\cdots>s_{k}>1$. Let $d_{i}(S)=s_{i}-s_{i+1}$ for all $i \in[k-1]$ and $d_{k}(S)=s_{k}-1$. Then

$$
\operatorname{cdes}_{n}(S)=\sum_{x_{1}, \ldots, x_{|S|} \in\{0,1\}}(-1)^{|S|-\sum_{j=1}^{|S|} x_{j}} \prod_{i=1}^{|S|}\left(1+\sum_{j=1}^{i} x_{i}\right)^{d_{i}(S)}
$$

for each positive integer $n$ and $S \subseteq[2, n]$.

Received November 19, 2018; Accepted January 15, 2019.

Communicated by Xuding Zhu.

2010 Mathematics Subject Classification. 05A15.

Key words and phrases. circular descent, generating tree, permutation, permutation tableaux.

*Corresponding author. 
The concept of generating trees has been introduced in the literature by Chung, Graham, Hoggat and Kleiman in [1] to examine Baxter permutations. There are closed relations between the generating trees and many combinatorial models. Also we describe a class of generating binary trees $T_{k}$ with weights. We find that the number of permutations in the set $\operatorname{CDES}_{n}(S)$ corresponds to the weights of $T_{k}$.

Postnikov [13 and Williams [16] mentioned the conceptions of permutation tableau when they studied the enumeration of the totally positive Grassmannian cells. Surprisingly, there are the closed connections between permutation tableaux and a statistical physics model called the Partially Asymmetric Exclusion Process 2, 4,5.

Following [15], we give the definition of the permutation tableau as follows. Recall that a partition $\lambda=\left(\lambda_{1}, \ldots, \lambda_{k}\right)$ is a weakly decreasing sequence of non-negative integers. For a partition $\lambda$, which $\sum \lambda_{i}=m$, the Young diagram $Y_{\lambda}$ of Shape $\lambda$ is a left-justified diagram of $m$ boxes, with $\lambda_{i}$ boxes in the $i$-th row.

Define a permutation tableau $\mathcal{T}_{n}^{k}$ to be a partition $\lambda$ such that $Y_{\lambda}$ is contained in a $k \times(n-k)$ rectangle, together with filling of the boxes of $Y_{\lambda}$ with 0 's and 1's such that the following properties hold:

(1) Each column of the rectangle contains at least one 1.

(2) There is no 0 which has a 1 above it in the same column and a 1 to its left in the same row.

The partition $\lambda$ is called the shape of the permutation tableau $\mathcal{T}_{n}^{k}$. Using a bijection $I$ in $[3$, as an application of the main results, we give the enumeration of permutation tableaux according to their shape.

A weak excedance of a permutation $\sigma$ is a value $\sigma(i)$ such that $\sigma(i) \geq i$. In this situation, the index $i$ (resp. $\sigma(i)$ ) is called a weak excedance bottom (resp. top) of $\sigma$. Obviously, non-weak excedance bottom and non-weak excedance top can be defined in terms of $i$ and $\sigma(i)$ such that $\sigma(i)<i$. Furthermore, for any $\sigma \in \mathfrak{S}_{n}$, the non-weak excedance bottom set of a permutation $\sigma$, denoted by $\operatorname{NWEXB}(\sigma)$, as $\operatorname{NWEXB}(\sigma)=\{i \mid$ $\sigma(i)<i\}$. For any $S \subseteq[n]$, define a set $\operatorname{NWEXB}_{n}(S)$ as $\operatorname{NWEXB}_{n}(S)=\left\{\sigma \in \mathfrak{S}_{n} \mid\right.$ $\operatorname{NWEXB}(\sigma)=S\}$ and use nwexb $\operatorname{mon}_{n}(S)$ to denote the number of the permutations in the set $\operatorname{NWEXB}_{n}(S)$, i.e., $\operatorname{nwexb}_{n}(S)=\left|\operatorname{NWEXB}_{n}(S)\right|$.

There are closed relation between $\operatorname{nwexb}_{n}(S)$ and the Genocchi numbers. The study of Genocchi numbers and their combinatorial interpretations has received much attention 6 12, 14]. Particularly, Michael Domartzki [6] give the definition of $k$-th generalized Genocchi numbers $\left\{G_{2 n}^{(k)}\right\}_{n \geq 1}$ as follows:

Let $A_{n+1}^{(k)}(X)$ be the following Gandhi polynomial in $X$ :

$$
A_{0}^{(k)}(X)=1 \quad \text { and } \quad A_{n+1}^{(k)}(X)=X^{k} A_{n}^{(k)}(X+1)-(X-1)^{k} A_{n}^{(k)}(X), \quad \forall n \geq 0 .
$$


Define $k$-th generalized Genocchi numbers $\left\{G_{2 n}^{(k)}\right\}_{n \geq 1}$ by $G_{2 n}^{(k)}=A_{n-1}^{(k)}(1)$. In [8], Dumont has given several interpretations of the usual Genocchi numbers $G_{2 n}^{(2)}$ in terms of permutations. Michael Domartzki [6] concluded that the number of permutations $\sigma$ of $[k n]$ such that $\sigma(i) \geq i$ if and only if $\sigma(i) \equiv 0(\bmod k)$ is equal to $G_{2 n+2}^{(k)}$. As another direct application of the main results in this paper, we give the enumeration of permutations by the non-weak excedance bottom set. This generalizes the results in [6].

We organize this paper as follows. In Section 2, we will give the proof of the main theorem. In Section 3, we will associate circular descent sets to a generating binary tree. In Section 4, we give some applications of the main theorem of this paper. We enumerate permutation tableaux according to their shape. We also counting the number of the permutations by the non-weak excedance bottom set.

\section{The proof of the main result}

In this section, we will give the proof of Theorem 1.1. We will derive some recursions and consider the generating function for the sequence $\operatorname{cdes}_{n}(S)$ as well. We start with some definitions. For any $\sigma \in \mathfrak{S}_{n}$, let $\tau=\sigma\left(i_{1}\right) \sigma\left(i_{2}\right) \cdots \sigma\left(i_{k}\right)$ be a subsequence of $\sigma$ and "red" be the increasing bijection from $\left\{\sigma\left(i_{1}\right), \sigma\left(i_{2}\right), \ldots, \sigma\left(i_{k}\right)\right\}$ to $[k]$. Define the reduction of the subsequence $\tau$, denote $\operatorname{red}(\tau)$, as $\operatorname{red}\left(\sigma\left(i_{1}\right)\right) \operatorname{red}\left(\sigma\left(i_{2}\right)\right) \cdots \operatorname{red}\left(\sigma\left(i_{k}\right)\right)$. For any $S \subseteq[2, n]$, define a set $\delta(S)$ as $\delta(S)=\{s-1 \mid s \in S\}$, clearly, $\delta(S) \subseteq[n-1]$. First, we easily obtain the following lemma.

Lemma 2.1. Let $n \geq 1$ and $S \subseteq[n]$. Then $\operatorname{CDES}_{n}(S) \neq \emptyset$ if and only if $1 \notin S$.

Proof. Note that it is impossible for the letter 1 to be a circular descent. For any $S \subseteq$ $[2, n]$, suppose that $S=\left\{i_{1}, \ldots, i_{k}\right\}$, where $i_{1}<i_{2}<\cdots<i_{k}$. Let $T=[n] \backslash S=$ $\left\{1, j_{1}, \ldots, j_{n-k-1}\right\}$, where $1<j_{1}<\cdots<j_{n-k-1}$. Then $\left(i_{k}, i_{k-1}, \ldots, i_{1}, 1, j_{1}, \ldots, j_{n-k-1}\right)$ $\in \operatorname{CDES}_{n}(S)$.

Lemma 2.2. Suppose that $n$ is a positive integer with $n \geq 2$ and $S \subseteq[2, n]$. Let $i=$ $\min (S)$. Then

$$
\operatorname{cdes}_{n}(S)=\operatorname{cdes}_{n}(S \cup\{i-1\} \backslash\{i\})+\operatorname{cdes}_{n-1}(\delta(S))+\operatorname{cdes}_{n-1}(\delta(S) \backslash\{i-1\}) .
$$

Proof. Let $T_{1}$ be a set of the permutations $\sigma$ in the set $\operatorname{CDES}_{n}(S)$ such that $i$ and $i-1$ are not adjacent in $\sigma$, i.e., $T_{1}=\left\{\sigma \in \operatorname{CDES}_{n}(S)|| \sigma^{-1}(i)-\sigma^{-1}(i-1) \mid \geq 2\right\}$. Let $T_{2}$ (resp. $T_{3}$ ) be a set of the permutations $\sigma$ in the set $\operatorname{CDES}_{n}(S)$ such that the position of $i-1$ (resp. $i$ ) is exactly the left of $i$ (resp. $i-1$ ), i.e., $T_{2}=\left\{\sigma \in \operatorname{CDES}_{n}(S) \mid \sigma^{-1}(i)=\right.$ $\left.\sigma^{-1}(i-1)+1\right\}\left(\right.$ resp. $\left.T_{3}=\left\{\sigma \in \operatorname{CDES}_{n}(S) \mid \sigma^{-1}(i-1)=\sigma^{-1}(i)+1\right\}\right)$. Furthermore, let $S_{1}=S \cup\{i-1\} \backslash\{i\}, S_{2}=\delta(S)$ and $S_{3}=\delta(S) \backslash\{i-1\}$. To obtain the results of the 
lemma, it is sufficient to give bijections from $T_{1}$ to $\operatorname{CDES}_{n}\left(S_{1}\right), T_{2}$ to $\operatorname{CDES}_{n-1}\left(S_{2}\right)$ and $T_{3}$ to $\mathrm{CDES}_{n-1}\left(S_{3}\right)$, respectively. We discuss the following two cases.

Case 1: The bijection from $T_{1}$ to $\operatorname{CDES}_{n}\left(S_{1}\right)$. For any $\sigma \in T_{1}$, we exchange the positions of $i$ and $i-1$ in $\sigma$ and denote the obtained permutation by $\tau$. Suppose that $\sigma(j)=i$ and $\sigma(k)=i-1$. Then $i-1 \notin S$ implies that either $\sigma(k+1)>i$ or $k=n$. It is easy to check $\sigma(j+1)<i-1$ since $i$ and $i-1$ are not adjacent in $\sigma$. Hence, the letter $i-1$ is a circular descent in $\tau$. Similarly, we may prove that it is impossible for the letter $i$ to be a circular descent in $\tau$. Obviously, this is a bijection.

Case 2: The bijection $\phi$ from $T_{2}$ to $\operatorname{CDES}_{n}\left(S_{2}\right)$. First, we claim that $T_{2}=\emptyset$ if $i=2$. Otherwise, the letter 2 can't be a circular descent since the position of 1 is exactly at the left of 2. Note that $2 \in S$ implies $1 \in S_{2}$. Lemma 2.1 tells us that $\operatorname{CDES}_{n-1}\left(S_{2}\right)=\emptyset$. When $i \geq 3$, we delete the letter $i$ from $\sigma$ and denote the obtained subsequence as $\tau$. Let $\phi(\sigma)=\operatorname{red}(\tau)$. Suppose $\sigma(j)=i$. Then $\sigma(j+1)<i-1$ since the position of $i-1$ is exactly at the left of $i$. Hence, $i-1$ is a circular descent of $\phi(\sigma)$ and $\phi(\sigma) \in \operatorname{CDES}_{n-1}\left(S_{2}\right)$. Conversely, for any $\tau \in \operatorname{CDES}_{n-1}\left(S_{2}\right)$, we increase each of the letters larger than $i-1$ by 1 and insert the letter $i$ behind $i-1$. Denote the obtained sequence as $\phi^{-1}(\tau)$. Suppose that $\tau(j)=i-1$. Then $\tau(j+1)<i-1$ since $i-1$ is a circular descent of $\tau$. This implies $\phi^{-1}(\tau) \in T_{2}$, hence, $\phi$ is a bijection from $T_{2}$ to $\operatorname{CDES}_{n}\left(S_{2}\right)$.

The bijection from $T_{3}$ to $\operatorname{CDES}_{n}\left(S_{3}\right)$ is similar to Case 2. The proof is completed.

Corollary 2.3. Suppose that $n$ is a positive integer with $n \geq 3$ and $S \subseteq[3, n]$. Then $\operatorname{cdes}_{n}(S \cup\{2\})=\operatorname{cdes}_{n-1}(\delta(S))$.

Proof. For any $\sigma \in \operatorname{CDES}_{n}(S \cup\{2\})$, the position of the letter 2 have to be exactly at the left of 1 in $\sigma$. Let $T_{3}$ be defined as that in $\operatorname{Lemma}_{2.2}$. Then $\operatorname{CDES}_{n}(S \cup\{2\})=T_{3}$. Hence, $\operatorname{cdes}_{n}(S \cup\{2\})=\operatorname{cdes}_{n-1}(\delta(S \cup\{2\}) \backslash\{1\})=\operatorname{cdes}_{n-1}(\delta(S))$.

Lemma 2.4. Suppose that $n$ is a positive integer with $n \geq 2$ and $S \subseteq[2, n]$. Let $j=$ $\max (S)$. Then

(1) $\operatorname{cdes}_{j}(S)=\operatorname{cdes}_{n}(S)$ for each integer $n \geq j$.

(2) $\operatorname{cdes}_{n}(\emptyset)=1$ for each positive integer $n$.

Proof. For any $\sigma \in \operatorname{CDES}_{n}(S)$, since the letters $j+1, \ldots, n$ are not circular descents of $\sigma$, we have $\sigma(k)=k$ for all $k \in[j+1, n]$. This $\operatorname{implies}_{\operatorname{cdes}_{n}}(S)=\operatorname{cdes}_{j}(S)$. Similarly, we have $\operatorname{cdes}_{n}(\emptyset)=1$.

Lemma 2.5. Suppose that $n$ is a positive integer with $n \geq 2$. Then $\operatorname{cdes}_{n}(\{n\})=2^{n-1}-1$. 
Proof. Let $A=\left\{\sigma(t): t<\sigma^{-1}(n)\right\}$ and $B=\left\{\sigma(t): t>\sigma^{-1}(n)\right\}$. Then $B \neq \emptyset$ since $n$ is the circular descent of $\sigma$. Furthermore, the elements of $A$ and $B$ form a increasing subsequence of $\sigma$ respectively since $n$ is the unique circular descent. For each letter $j \neq n$, the position of $j$ has two possibilities at the left or right of $n$. Therefore, $\operatorname{cdes}_{n}(\{n\})=$ $2^{n-1}-1$ since $B \neq \emptyset$.

In the next lemma, we will derive another recursion for the sequence $\operatorname{cdes}_{n}(S)$.

Lemma 2.6. Suppose that $n$ is a positive integer with $n \geq 3$ and $S \subseteq[2, n-1]$. Then

$$
\operatorname{cdes}_{n}(S \cup\{n\})=(n-1-|S|) \operatorname{cdes}_{n-1}(S)+\sum_{i \in[n-1] \backslash S} \operatorname{cdes}_{n-1}(S \cup\{i\}) .
$$

Proof. It is clear that $i<n$. For each $\sigma \in \mathfrak{S}_{n-1}$, we may extend $\sigma$ to be a permutation of $\mathfrak{S}_{n}$ by inserting $n$ into $\sigma$. It is easy to see that there are $n-1-|S|$ ways to insert $n$ such that the set of circular descent of the new permutation is $S \cup\{n\}$. For any $i \in[n-1] \backslash S$ and $\sigma \in \operatorname{CDES}_{n-1}(S \cup\{i\})$, if we place the letter $n$ after $i$ then the resulting permutation has the set $S \cup\{n\}$. So we have $\operatorname{cdes}_{n}(S \cup\{n\})=(n-1-|S|) \operatorname{cdes}_{n-1}(S)+$ $\sum_{i \in[n-1] / S} \operatorname{cdes}_{n-1}(S \cup\{i\})$.

For any $S \in[2, n]$, suppose $S=\left\{i_{1}, i_{2}, \ldots, i_{k}\right\}$, let $\mathbf{x}_{S}$ stand for the monomial $x_{i_{1}-1} x_{i_{2}-1} \cdots x_{i_{k}-1}$; particularly, let $\mathbf{x}_{\emptyset}=1$. Given $n \geq 2$, we define a generating function

$$
g_{n}\left(x_{1}, x_{2}, \ldots, x_{n-1} ; y\right)=\sum_{\sigma \in \mathfrak{S}_{n}} \mathbf{x}_{\operatorname{CDES}(\sigma)} y^{|\operatorname{CDES}(\sigma)|}
$$

We also write $g_{n}\left(x_{1}, x_{2}, \ldots, x_{n-1} ; y\right)$ as $g_{n}$ for short. We call it circular descent polynomial.

Corollary 2.7. Let $n$ be a positive integer with $n \geq 2$ and $g_{n}=\sum_{\sigma \in \mathfrak{S}_{n}} \mathbf{x}_{\operatorname{CDES}(\sigma)} y^{|\operatorname{CDES}(\sigma)|}$. Then $g_{n}$ satisfies the following recursion:

$$
g_{n+1}=\left(1+n x_{n} y\right) g_{n}+x_{n} \sum_{i=1}^{n-1} \frac{\partial g_{n}}{\partial x_{i}}-x_{n} y^{2} \frac{\partial g_{n}}{\partial y}
$$

for all $n \geq 2$ with initial condition $g_{2}=1+x_{1} y$, where the notation $\partial g_{n} / \partial y$ denotes partial differentiation of $g_{n}$ with respect to $y$.

Proof. Obviously, $g_{2}=1+x_{1} y$ and $\sum_{\sigma \in \mathfrak{S}_{n}} \mathbf{x}_{\operatorname{CDES}(\sigma)} y^{|\operatorname{CDES}(\sigma)|}=\sum_{S \subseteq[2, n]} \operatorname{cdes}_{n}(S) \mathbf{x}_{S} y^{|S|}$. Hence,

$$
\begin{aligned}
g_{n+1} & =\sum_{S \subseteq[2, n+1]} \operatorname{cdes}_{n+1}(S) \mathbf{x}_{S} y^{|S|} \\
& =\sum_{S \subseteq[2, n+1], n+1 \in S} \operatorname{cdes}_{n+1}(S) \mathbf{x}_{S} y^{|S|}+\sum_{S \subseteq[2, n+1], n+1 \notin S} \operatorname{cdes}_{n+1}(S) \mathbf{x}_{S} y^{|S|}
\end{aligned}
$$




$$
\begin{aligned}
= & \sum_{S \subseteq[2, n]}\left[(n-|S|) \operatorname{cdes}_{n}(S)+\sum_{i \in[2, n] \backslash S} \operatorname{cdes}_{n}(S \cup\{i\})\right] \mathbf{x}_{S} x_{n} y^{|S|+1}+g_{n} \\
= & \sum_{S \subseteq[2, n]} \sum_{i \in[2, n] \backslash S} \operatorname{cdes}_{n}(S \cup\{i\}) \mathbf{x}_{S} x_{n} y^{|S|+1} \\
& -\sum_{S \subseteq[2, n]}|S| \operatorname{cdes}_{n}(S) \mathbf{x}_{S} x_{n} y^{|S|+1}+\left(1+n x_{n} y\right) g_{n} .
\end{aligned}
$$

Note that

$$
\frac{\partial g_{n}}{\partial y}=\sum_{S \subseteq[2, n]}|S| \operatorname{cdes}_{n}(S) \mathbf{x}_{S} y^{|S|-1}
$$

and

$$
\begin{aligned}
\sum_{S \subseteq[2, n]} \sum_{i \in[2, n] \backslash S} \operatorname{cdes}_{n}(S \cup\{i\}) \mathbf{x}_{S} x_{n} y^{|S|+1} & =\sum_{S \subseteq[2, n], S \neq \emptyset} \operatorname{cdes}_{n}(S) x_{n} y^{|S|} \sum_{i \in S} \frac{\mathbf{x}_{S}}{x_{i-1}} \\
& =x_{n} \sum_{i=1}^{n-1} \frac{\partial g_{n}}{\partial x_{i}} .
\end{aligned}
$$

Therefore,

$$
g_{n+1}=\left(1+n x_{n} y\right) g_{n}+x_{n} \sum_{i=1}^{n-1} \frac{\partial g_{n}}{\partial x_{i}}-x_{n} y^{2} \frac{\partial g_{n}}{\partial y}
$$

By computer search, we obtain the values of $\operatorname{cdes}_{n}(S)$ for all $2 \leq n \leq 5$ and $S \subseteq[2, n]$. Here we list the generating function $g_{n}$ for $2 \leq n \leq 5$ :

$$
\begin{aligned}
g_{2}= & 1+x_{1} y, \\
g_{3}= & 1+x_{1} y+3 x_{2} y+x_{1} x_{2} y^{2}, \\
g_{4}= & 1+x_{1} y+3 x_{2} y+x_{1} x_{2} y^{2}+7 x_{3} y+3 x_{1} x_{3} y^{2}+7 x_{2} x_{3} y^{2}+x_{1} x_{2} x_{3} y^{3}, \\
g_{5}= & 1+x_{1} y+3 x_{2} y+x_{1} x_{2} y^{2}+7 x_{3} y+3 x_{1} x_{3} y^{2}+7 x_{2} x_{3} y^{2}+x_{1} x_{2} x_{3} y^{3} \\
& +15 x_{4} y+7 x_{1} x_{4} y^{2}+17 x_{2} x_{4} y^{2}+3 x_{1} x_{2} x_{4} y^{3}+31 x_{3} x_{4} y^{2} \\
& +7 x_{1} x_{3} x_{4} y^{3}+15 x_{2} x_{3} x_{4} y^{3}+x_{1} x_{2} x_{3} x_{4} y^{4}
\end{aligned}
$$

Proof of Theorem 1.1. Let $k=|S|$. First, by induction on $k$. For convenience, we let

$$
R_{k}\left(S ; x_{1}, \ldots, x_{k}\right)=(-1)^{k-x_{1}-\cdots-x_{k}} \prod_{j=1}^{k}\left(1+x_{1}+\cdots+x_{j}\right)^{d_{j}(S)} .
$$

By Lemma 2.4(1), we may always suppose $n \in S$. When $k=1$,

$$
\operatorname{cdes}_{n}(S)=\sum_{x_{1} \in\{0,1\}}(-1)^{1-x_{1}}\left(1+x_{1}\right)^{n-1}=2^{n-1}-1 .
$$


By Lemma 2.5, the theorem holds when $k=1$. Now, assume that the theorem holds for $k^{\prime} \leq k$, i.e.,

$$
\operatorname{cdes}_{n}(S)=\sum_{x_{1}, \ldots, x_{k^{\prime}} \in\{0,1\}} R_{k^{\prime}}\left(S ; x_{1}, \ldots, x_{k^{\prime}}\right) .
$$

We consider the case with $k^{\prime}=k+1$. If $s_{k+1}=2$, then let $S^{\prime}=S \backslash\left\{s_{k+1}\right\}$. Corollary 2.3 tells us that $\operatorname{cdes}_{n}(S)=\operatorname{cdes}_{n}\left(S^{\prime} \cup\{2\}\right)=\operatorname{cdes}_{n-1}\left(\delta\left(S^{\prime}\right)\right)$. Furthermore, we have $d_{j}\left(\delta\left(S^{\prime}\right)\right)=d_{j}(S)$ for any $j \in[k]$ and $d_{k+1}(S)=1$. Note that $\left|\delta\left(S^{\prime}\right)\right|=k$. So,

$$
\begin{aligned}
& R_{k+1}\left(S ; x_{1}, \ldots, x_{k}, 1\right)=R_{k}\left(\delta\left(S^{\prime}\right) ; x_{1}, \ldots, x_{k}\right)\left(2+\sum_{i=1}^{k} x_{i}\right) \\
& R_{k+1}\left(S ; x_{1}, \ldots, x_{k}, 0\right)=-R_{k}\left(\delta\left(S^{\prime}\right) ; x_{1}, \ldots, x_{k}\right)\left(1+\sum_{i=1}^{k} x_{i}\right) .
\end{aligned}
$$

Therefore,

$$
R_{k+1}\left(S ; x_{1}, \ldots, x_{k}, 1\right)+R_{k+1}\left(S ; x_{1}, \ldots, x_{k}, 0\right)=R_{k}\left(\delta\left(S^{\prime}\right) ; x_{1}, \ldots, x_{k}\right)
$$

By the induction hypothesis, we have

$$
\operatorname{cdes}_{n}\left(\delta\left(S^{\prime}\right)\right)=\sum_{x_{1}, \ldots, x_{k} \in\{0,1\}} R_{k}\left(\delta\left(S^{\prime}\right) ; x_{1}, \ldots, x_{k}\right) .
$$

Hence,

$$
\begin{aligned}
\operatorname{cdes}_{n}(S) & =\operatorname{cdes}_{n}\left(\delta\left(S^{\prime}\right)\right) \\
& =\sum_{x_{1}, \ldots, x_{k} \in\{0,1\}} R_{k}\left(\delta\left(S^{\prime}\right) ; x_{1}, \ldots, x_{k}\right) \\
& =\sum_{x_{1}, \ldots, x_{k} \in\{0,1\}}\left[R_{k+1}\left(S ; x_{1}, \ldots, x_{k}, 1\right)+R_{k+1}\left(S ; x_{1}, \ldots, x_{k}, 0\right)\right] \\
& =\sum_{x_{1}, \ldots, x_{k+1} \in\{0,1\}} R_{k+1}\left(S ; x_{1}, \ldots, x_{k+1}\right) .
\end{aligned}
$$

So, the theorem holds for $k^{\prime}=k+1$ and $\min S=2$.

Let $i=\min S$. Now, by induction on $i$ again. Suppose the theorem holds for $k^{\prime}=k+1$, $i^{\prime}=\min S^{\prime}$ and $2 \leq i^{\prime} \leq i$. Suppose $\min S=i+1$. Let the sets $S_{j}$ be defined as that in Lemma 2.2 for $j=1,2,3$. It is easy to obtain that $d_{j}\left(S_{1}\right)=d_{j}\left(S_{2}\right)=d_{j}\left(S_{3}\right)=d_{j}(S)$ for any $j \in[k-1], d_{k}\left(S_{1}\right)=d_{k}(S)+1, d_{k+1}\left(S_{1}\right)=d_{k+1}\left(S_{2}\right)=d_{k+1}(S)-1, d_{k}\left(S_{2}\right)=d_{k}(S)$, and $d_{k}\left(S_{3}\right)=d_{k}(S)+d_{k+1}(S)-1$. Hence, we have

$$
R_{k+1}\left(S_{1} ; x_{1}, \ldots, x_{k+1}\right)=R_{k+1}\left(S ; x_{1}, \ldots, x_{k+1}\right)\left(1+\sum_{j=1}^{k} x_{j}\right)\left(1+\sum_{j=1}^{k+1} x_{j}\right)^{-1}
$$




$$
\begin{aligned}
R_{k+1}\left(S_{2} ; x_{1}, \ldots, x_{k+1}\right) & =R_{k+1}\left(S ; x_{1}, \ldots, x_{k+1}\right)\left(1+\sum_{j=1}^{k+1} x_{j}\right)^{-1} \\
R_{k}\left(S_{3} ; x_{1}, \ldots, x_{k}\right) & =-\left(1+\sum_{j=1}^{k} x_{j}\right)^{-1} R_{k+1}\left(S ; x_{1}, \ldots, x_{k}, 0\right) .
\end{aligned}
$$

Note that

$$
\begin{aligned}
& R_{k+1}\left(S ; x_{1}, \ldots, x_{k+1}\right)-R_{k+1}\left(S_{1} ; x_{1}, \ldots, x_{k+1}\right)-R_{k+1}\left(S_{2} ; x_{1}, \ldots, x_{k+1}\right) \\
= & R_{k+1}\left(S_{1} ; x_{1}, \ldots, x_{k+1}\right)\left[1-\left(1+\sum_{j=1}^{k} x_{j}\right)\left(1+\sum_{j=1}^{k+1} x_{j}\right)^{-1}-\left(1+\sum_{j=1}^{k+1} x_{j}\right)^{-1}\right] \\
= & \frac{x_{k+1}-1}{1+x_{1}+\cdots+x_{k+1}} R_{k+1}\left(S ; x_{1}, \ldots, x_{k+1}\right) .
\end{aligned}
$$

Since $\min \left(S_{1}\right)=\min \left(S_{2}\right)=i,\left|S_{3}\right|=k$, by the induction hypothesis, we have

$$
\begin{aligned}
\operatorname{cdes}_{n}\left(S_{1}\right) & =\sum_{x_{1}, \ldots, x_{k+1} \in\{0,1\}} R_{k+1}\left(S_{1} ; x_{1}, \ldots, x_{k+1}\right), \\
\operatorname{cdes}_{n-1}\left(S_{2}\right) & =\sum_{x_{1}, \ldots, x_{k+1} \in\{0,1\}} R_{k+1}\left(S_{2} ; x_{1}, \ldots, x_{k+1}\right), \\
\operatorname{cdes}_{n-1}\left(S_{3}\right) & =\sum_{x_{1}, \ldots, x_{k} \in\{0,1\}} R_{k}\left(S_{3} ; x_{1}, \ldots, x_{k}\right) .
\end{aligned}
$$

It is easy to check that

$$
\begin{aligned}
& \sum_{x_{1}, \ldots, x_{k+1} \in\{0,1\}} R_{k+1}\left(S ; x_{1}, \ldots, x_{k+1}\right)-\operatorname{cdes}_{n}\left(S_{1}\right)-\operatorname{cdes}_{n-1}\left(S_{2}\right) \\
= & \sum_{x_{1}, \ldots, x_{k+1} \in\{0,1\}} \frac{x_{k+1}-1}{1+x_{1}+\cdots+x_{k+1}} R_{k+1}\left(S ; x_{1}, \ldots, x_{k+1}\right) \\
= & \sum_{x_{1}, \ldots, x_{k} \in\{0,1\}}-\frac{1}{1+x_{1}+\cdots+x_{k}} R_{k+1}\left(S ; x_{1}, \ldots, x_{k}, 0\right) \\
= & \sum_{x_{1}, \ldots, x_{k} \in\{0,1\}} R_{k}\left(S_{3} ; x_{1}, \ldots, x_{k}\right) \\
= & \operatorname{cdes}_{n-1}\left(S_{3}\right) .
\end{aligned}
$$

So, $\sum_{x_{1}, \ldots, x_{k+1} \in\{0,1\}} R_{k+1}\left(S ; x_{1}, \ldots, x_{k+1}\right)=\operatorname{cdes}_{n}\left(S_{1}\right)+\operatorname{cdes}_{n-1}\left(S_{2}\right)+\operatorname{cdes}_{n-1}\left(S_{3}\right)$. By Lemma 2.2, we have $\operatorname{cdes}_{n}(S)=\operatorname{cdes}_{n}\left(S_{1}\right)+\operatorname{cdes}_{n-1}\left(S_{2}\right)+\operatorname{cdes}_{n-1}\left(S_{3}\right)$. Hence,

$$
\operatorname{cdes}_{n}(S)=\sum_{x_{1}, \ldots, x_{k+1} \in\{0,1\}} R_{k+1}\left(S ; x_{1}, \ldots, x_{k+1}\right) .
$$

This complete the proof. 


\section{Circular descent sets and generating trees}

In this section we will associate circular descent sets to a generating binary tree. We consider the generating binary tree as follows:

$$
\text { root }:(1) \text { and rule }:(k) \rightarrow(k)(k+1) \text {. }
$$

This means first that the root has label (1), and then for all $k$, any node labeled $(k)$ will have 2 descendants and they will have the labels $(k),(k+1)$. We denote the obtained tree by $T$. For any vertex $v$, there exists a unique path $P_{v}$ connecting $v$ and the root. Define the height of $v$ to be the number of the edges in $P_{v}$. If $\{w, v\}$ is an edge of $T$ and the height of a vertex $w$ is less than the height of $v$, then we say $w$ is the father of $f$ (resp. $v$ is a child of $w$ ), denoted by $w=f(v)$ (resp. $v \in c(w))$.

Suppose $E(T)$ is the edge set of $T$. Define the edge weight function of $T$, denoted $e w$, as $e w(\{w, v\})=(-1)^{1-k+l}$, where $(k)$ and $(l)$ are the labels of the vertices $v$ and $w$ respectively, for any $\{w, v\} \in E(T)$ with $w=f(v)$. Clearly, $k-l \in\{0,1\}$. Given an infinite sequence $\vec{d}=\left(d_{1}, d_{2}, \ldots, d_{j}, \ldots\right)$ of integers, we define the vertex weight function of $T$, denoted $v w_{\vec{d}}$, as $v w_{\vec{d}}(v)=k^{d_{j}}$, where $(k)$ is the labels of the vertex $v$ and $j$ is the height of $v$, for any non-root vertex $v$, and $v w(v)=1$ if $v$ is the root. For any $v \in V(T)$, recall that there exists a unique path $P_{v}$ connecting $v$ and the root. We define the weight $\omega_{\vec{d}}\left(P_{v}\right)$ of $P_{v}$ as $\omega_{\vec{d}}\left(P_{v}\right)=\prod_{u \in V\left(P_{v}\right)} v w_{\vec{d}}(u) \prod_{e \in E\left(P_{v}\right)} e w(e)$. For a fixed $k \geq 1$, let $T_{k}$ be the subgraph of $T$ induced by the vertices with height less or equal to $k$ and $\operatorname{Leaf}\left(T_{k}\right)$ the set of the leaves of $T_{k}$. Define the wight $\omega_{\vec{d}}\left(T_{k}\right)$ of $T_{k}$ as follows:

$$
\omega_{\vec{d}}\left(T_{k}\right)=\sum_{v \in \operatorname{Leaf}\left(T_{k}\right)} \omega_{\vec{d}}\left(P_{v}\right)=\sum_{v \in \operatorname{Leaf}\left(T_{k}\right)} \prod_{u \in V\left(P_{v}\right)} v w_{\vec{d}}(u) \prod_{e \in E\left(P_{v}\right)} e w(e) .
$$

Theorem 3.1. Let $k \geq 1$ and $\vec{d}=\left(d_{1}, \ldots, d_{k}\right)$ be a sequence of nonnegative integers. Then

$$
\omega_{\vec{d}}\left(T_{k}\right)=\sum_{x_{1}, x_{2}, \ldots, x_{k} \in\{0,1\}}(-1)^{k-\sum_{i=1}^{k} x_{i}} \prod_{i=0}^{k}\left(1+\sum_{j=1}^{i} x_{j}\right)^{d_{i}} .
$$

Proof. Use $D$ to denote the set of all the $k$-tuples $\left(x_{1}, \ldots, x_{k}\right)$ such that $x_{i} \in\{0,1\}$. First, we establish a bijection $\theta$ from Leaf $\left(T_{k}\right)$ to $D$. For any $v \in \operatorname{Leaf}\left(T_{k}\right)$, let $P_{v}$ be the unique path connecting $v$ and the root. Suppose that $P_{v}=w_{0} w_{1} \cdots w_{k}$. Then $w_{0}$ is the root and $w_{k}=v$. Let $x_{i}$ be the difference of the labels of $w_{i}$ and $w_{i-1}$ for any $i \in[k]$ and $\theta(v)=\left(x_{1}, \ldots, x_{k}\right)$. It is easy to obtain $x_{i} \in[0,1]$ for any $i \in[k]$ by the construction of the generating binary tree $T$. Therefore, $\theta(v) \in D$. Conversely, for any $\left(x_{1}, \ldots, x_{k}\right) \in D$, let $w_{0}=1$ and the label of $w_{i}$ is the sum of the label of $w_{i-1}$ and $x_{i}$ for any $i \in[k]$. Equivalently, the label of $w_{i}$ is $1+\sum_{j=1}^{i} x_{i}$ for all $i \in[0, k]$. So, 
$\theta^{-1}\left(\left(x_{1}, \ldots, x_{k}\right)\right)=w_{k} \in \operatorname{Leaf}\left(T_{k}\right)$. Hence, $\theta$ is a bijection from Leaf $\left(T_{k}\right)$ to $D$. Moreover, for any $v \in \operatorname{Leaf}\left(T_{k}\right)$, we have

$$
\begin{aligned}
\omega_{\vec{d}}\left(P_{v}\right) & =\prod_{u \in V\left(P_{v}\right)} v w_{\vec{d}}(u) \prod_{e \in E\left(P_{v}\right)} e w(e) \\
& =\prod_{i=1}^{k}\left(1+\sum_{j=1}^{i} x_{j}\right)^{d_{i}} \prod_{i=1}^{k}(-1)^{1-x_{i}} \\
& =(-1)^{k-\sum_{i=1}^{k} x_{i}} \prod_{i=1}^{k}\left(1+\sum_{j=1}^{i} x_{j}\right)^{d_{i}},
\end{aligned}
$$

where $\left(x_{1}, \ldots, x_{k}\right)=\theta(v)$. Hence,

$$
\omega_{\vec{d}}\left(T_{k}\right)=\sum_{x_{1}, x_{2}, \ldots, x_{k} \in[0,1]}(-1)^{k-\sum_{i=1}^{k} x_{i}} \prod_{i=1}^{k}\left(1+\sum_{j=1}^{i} x_{j}\right)^{d_{i}} .
$$

Corollary 3.2. Suppose that $n \geq 2$ and $S \subseteq[2, n]$. Let $\vec{d}=\left(d_{1}(S), \ldots, d_{|S|}(S)\right)$. Then $\omega_{\vec{d}}\left(T_{|S|}\right)=\operatorname{cdes}_{n}(S)$.

For each $k \leq n$, consider the polynomial $g_{n, k}(\mathbf{x})=\sum_{\sigma \in \mathfrak{S}_{n},\left|\operatorname{CDES}_{n}(\sigma)\right|=k} \mathbf{x}_{\operatorname{CDES}_{n}(\sigma)}$. We call it the circular descent polynomial with size $k$. It is clear that $g_{n}=\sum_{i=0}^{k} g_{n, k} y^{k}$. Let $\mathscr{D}_{k, n}=\left\{\left(d_{1}, \ldots, d_{k}\right): d_{1}+\cdots+d_{k}=n-1, d_{i}>0\right.$ for all $\left.i \in[k]\right\}$. We define $\tau$ is a mapping from $\mathscr{D}_{k, n}$ to $[2, n]$ as $\tau(D)=\left\{1+d_{1}, \ldots, 1+d_{1}+\cdots+d_{k}\right\}$ for each $D \in \mathscr{D}_{k, n}$. We have the following corollary.

Corollary 3.3. $g_{n, k}(\mathbf{x})=\sum_{D \in \mathscr{D}_{n, k}} \omega\left(T_{k}\right) \mathbf{x}_{\tau(D)}$ and $g_{n}(\mathbf{x}, y)=\sum_{k=0}^{n}\left(\sum_{D \in \mathscr{D}_{n, k}} \omega\left(T_{k}\right)\right.$ $\left.\mathbf{x}_{\tau(D)}\right) y^{k}$.

In the final of this section, we will give a example to show the relation between $\operatorname{cdes}_{n}(S)$ and $\left.\omega_{\vec{d}}\left(T_{k}\right)\right)$. Suppose $k=2, n=1+d_{1}+d_{2}, d=\left\{d_{1}, d_{2}\right\}$ and $S=\left\{1+d_{1}, 1+d_{1}+d_{2}\right\}$. The weight $\omega_{d}\left(T_{2}\right)$ with respect to $d$ is Figure 3.1 . Therefore, $\omega_{\vec{d}}\left(T_{2}\right)=3^{d_{2}} 2^{d_{1}}-2^{d_{2}+d_{1}}-$ $2^{d_{2}} 1^{d_{1}}+1^{d_{1}+d_{2}}$. By Theorem 1.1, we have $\omega_{\vec{d}}\left(T_{2}\right)=\operatorname{cdes}_{n}(S)$.

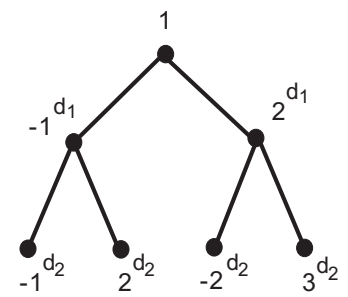

Figure 3.1: The weight of the generating binary tree $T_{2}$. 


\section{Some applications of the main theorem}

In this section, we will counting the number of permutation tableaux according to their shape and give the enumeration of permutations by the non-weak excedance bottom set.

For any $S \subseteq[2, n]$, define the type of the set $S$, denoted type $(S)$, as $\left(r_{1}^{m_{1}}, r_{2}^{m_{2}}, \ldots, r_{s}^{m_{s}}\right)$ if $S=\bigcup_{i=1}^{s}\left[r_{i}-m_{i}+1, r_{i}\right]$ such that $r_{i} \leq r_{i-1}-m_{i-1}-1$ for all $i \geq 2$. Obviously, $\sum_{i=1}^{s} m_{i}=|S|, r_{1}=\max S$ and $r_{s}-m_{s}+1=\min S$. We restate Theorem 1.1 as follows.

Lemma 4.1. Let $n$ be a positive integer with $n \geq 2$ and $S \subseteq[2, n]$. Suppose that type $(S)=$ $\left(r_{1}^{m_{1}}, r_{2}^{m_{2}}, \ldots, r_{s}^{m_{s}}\right)$ and let $r_{s+1}=1$ and $M_{i}=m_{1}+\cdots+m_{i}$ for any $i \in[s]$. Then

$$
\operatorname{cdes}_{n}(S)=\sum_{x_{1}, \ldots, x_{|S|} \in\{0,1\}}(-1)^{|S|-\sum_{j=1}^{|S|} x_{j}} \prod_{i=1}^{|S|}\left(1+\sum_{j=1}^{i} x_{j}\right) \prod_{i=1}^{s}\left(1+\sum_{j=1}^{M_{i}} x_{j}\right)^{r_{i}-r_{i+1}-m_{i}}
$$

Suppose that the partition $Y_{\lambda}$ is contained in the $k \times(n-k)$ rectangle. Regard the south-east border of $Y_{\lambda}$ as giving a path $\mathcal{P}=\left\{P_{i}\right\}_{i=1}^{n}$ of length $n$ from the northeast corner of the rectangle to the southwest corner the rectangle: label each of the steps in this path with a number from 1 to $n$ according to the order in which the step was taken. Sylvie Corteel and Philippe Nadeau [3] established a bijection $I$ between permutation tableaux of length $n$ and permutations of $[n]$. We state as the following lemma.

Lemma 4.2. [3] There is a bijection $\Phi$ from permutation tableaux of length $n$ to $\mathfrak{S}_{n}$. Moreover, for any permutation tableau $T_{n}^{k}$, the circular descent of $\sigma=\Phi\left(T_{n}^{k}\right)$ are precisely the labels on the horizontal edges of $\mathcal{P}$.

For any a partition $\lambda=\left(\lambda_{1}, \ldots, \lambda_{k}\right)$, there exists a unique strictly decreasing sequence $a_{\lambda}=\left(a_{1}, \ldots, a_{s}\right)$ with $s \leq k$ such that there are some $j$ satisfying $a_{j}=\lambda_{i}$ for any $i \in[k]$. On the other hand, let $b_{\lambda}=\left(b_{1}, \ldots, b_{s}\right)$ such that $b_{i}=\left|\left\{j \in[k] \mid \lambda_{j} \geq a_{i}\right\}\right|$ for all $i \in[s]$. The pair $\left(a_{\lambda}, b_{\lambda}\right)$ is called the type of the partition $\lambda$, denoted by type $(\lambda)$.

Corollary 4.3. Fix a partition $\lambda=\left(\lambda_{1}, \ldots, \lambda_{k}\right)$ with $\lambda_{k} \geq 1$. Suppose that type $(\lambda)=$ $\left(a_{\lambda}, b_{\lambda}\right), b_{0}=1$ and the length of $a_{\lambda}$ is $s$. Then the number of permutation tableaux $\mathcal{T}_{n}^{k}$ with shape $\lambda$ is equal to

$$
\sum_{x_{1}, \ldots, x_{n-k} \in\{0,1\}}(-1)^{n-k-\sum_{j=1}^{n-k} x_{j}} \prod_{i=1}^{n-k}\left(1+\sum_{j=1}^{i} x_{j}\right) \prod_{i=1}^{s}\left(1+\sum_{j=1}^{a_{s+1-i}} x_{j}\right)^{b_{s+1-i}-b_{s-i}} .
$$

Proof. Let $\mathcal{T}_{n}^{k}$ be a permutation tableau with shape $\lambda$. Since the type of $\lambda$ is $\left(a_{\lambda}, b_{\lambda}\right)$, let $a_{s+1}=0$, all the labels of the horizontal edges of $\mathcal{P}$ are in the set $S=\bigcup_{i=1}^{s}\left[a_{1}-a_{i}+b_{i}+\right.$ $\left.1, a_{1}-a_{i+1}+b_{i}\right]$. Clearly, $|S|=\lambda_{1}=a_{1}=n-k$. Let $m_{i}=a_{s+1-i}-a_{s+2-i}$ and $r_{i}=$ $a_{1}-a_{s+2-i}+b_{s+1-i}$ for all $1 \leq i \leq s$. It is easy to check that type $(S)=\left(r_{1}^{m_{1}}, r_{2}^{m_{2}}, \ldots, r_{s}^{m_{s}}\right)$. 
From Lemma 4.2 , it follows that the number of permutation tableaux $T_{n}^{k}$ with shape $\lambda$ is equal to $\operatorname{cdes}_{n}(S)$. Lemma 4.1 implies that the number of permutation tableaux $T_{n}^{k}$ with shape $\lambda$ is

$$
\sum_{x_{1}, \ldots, x_{n-k} \in\{0,1\}}(-1)^{n-k-\sum_{j=1}^{n-k} x_{j}} \prod_{i=1}^{n-k}\left(1+\sum_{j=1}^{i} x_{j}\right) \prod_{i=1}^{s}\left(1+\sum_{j=1}^{a_{s+1-i}} x_{j}\right)^{b_{s+1-i}-b_{s-i}} .
$$

We also need the following lemma [15].

Lemma 4.4. 15] There is a bijection $\Psi$ from permutation tableaux of length $n$ to $\mathfrak{S}_{n}$. Moreover, for any permutation tableau $\mathcal{T}_{n}^{k}$, the non-weak excedance bottoms of $\sigma=\Psi\left(\mathcal{T}_{n}^{k}\right)$ are precisely the labels on the horizontal edges of $\mathcal{P}$.

Combining Lemmas 4.2 and 4.4 , we immediately obtain the following corollary.

Corollary 4.5. Let $n$ be a positive integer with $n \geq 2$ and $S \subseteq[2, n]$. Then $\operatorname{nwexb}_{n}(S)=$ $\operatorname{cdes}_{n}(S)$ and $\sum_{\sigma \in \mathfrak{S}_{n}} \mathbf{x}_{\mathrm{NWEXB}_{n}(\sigma)} y^{\left|\operatorname{NWEXB}_{n}(\sigma)\right|}=\sum_{\sigma \in \mathfrak{S}_{n}} \mathbf{x}_{\mathrm{CDES}_{n}(\sigma)} y^{\left|\operatorname{CDES}_{n}(\sigma)\right|}$.

\section{Acknowledgments}

This work was supported by National Natural Science Foundation of China (Grants No. 11571235). The authors are thankful to the referees for their helpful comments to improve the paper.

\section{References}

[1] F. R. K. Chung, R. L. Graham, V. E. Hoggat, Jr. and M. Kleiman, The number of Baxter permutations, J. Combin. Theory Ser. A 24 (1978), no. 3, 382-394.

[2] S. Corteel, Crossings and alignments of permutations, Adv. in Appl. Math. 38 (2007), no. $2,149-163$.

[3] S. Corteel and P. Nadeau, Bijections for permutaion tableaux, European J. Combin. 30 (2009), no. 1, 295-310.

[4] S. Corteel and L. K. Williams, Permutation tableaux and the asymmetric exclusion process, Adv. in Appl. Math. 39 (2006), no. 3, 293-310.

[5] _ A Markov chain on permutations which projects to the PASEP, Int. Math. Res. Not. IMRN 2007 (2007), no. 17, Art. ID rnm055, 27 pp.

[6] M. Domaratzki, Combinatorial Interpretations of a generalization of the Genocchi numbers, J. Integer Seq. 7 (2004), no. 3, Article 04.3.6, 11 pp. 
[7] D. Dumont, Sur une conjecture de Gandhi concernant les nombres de Genocchi, Discrete Math. 1 (1972), no. 4, 321-327.

[8] _ Interprétations combinatoires des nombres de Genocchi, Duke Math. J. 41 (1974), 305-318.

[9] D. Dumont and D. Foata, Une propriété de symétrie des nombres de Genocchi, Bull. Soc. Math. France 104 (1976), no. 4, 433-451.

[10] D. Dumont and A. Randrianarivony, Dérangements et nombres de Genocchi, Discrete Math. 132 (1994), no. 1-3, 37-49.

[11] D. Dumont and G. Viennot, A combinatorial interpretation of the Seidel generation of Genocchi numbers, Ann. Discrete. Math. 6 (1980), 77-87.

[12] J. M. Gandhi, A conjectured representation of Genocchi numbers, Amer. Math. Monthly 77 (1970), no. 5, 505-506.

[13] A. Postnikov, Total positivity, Grassmannians, and networks, arXiv:math/0609764.

[14] J. Riordan and P. R. Stein, Proof of a conjecture on Genocchi numbers, Discrete Math. 5 (1973), 381-388.

[15] E. Steingrimsson and L. K. Willams, Permutation tableaux and permutation patterns, J. Combin. Theory Ser. A 114 (2007), no. 2, 211-234.

[16] L. K. Williams, Enumeration of totally positive Grassmann cells, Adv. Math. 190 (2005), no. 2, 319-342.

Hungyung Chang

Institute of Mathematics, Academia Sinica, Taipei, Taiwan

E-mail address: primesnark@gmail.com

Jun Ma

School of Mathematical Sciences, Shanghai Jiao Tong University, Shanghai, China E-mail address: majun904@sjtu.edu.cn

Jean Yeh

Department of Mathematics, Texas A\&M University, College Station, Texas, USA E-mail address: jeanyeh@math.tamu.edu 